\title{
Traumatismes et rituels du nationalisme flamand
}

\author{
Éclairage psycho-social d'anecdotes choisies de la \\ crise politique belge
}

Stéphane Baele

\section{Résumé/Abstract}

[Français] Comme l'ironisait le célèbre politologue Robert Jervis, se passer de la psychologie pour comprendre un événement politique revient à effectuer une «recherche d'ivrogne »; tel l'ivrogne qui cherche ses clefs là où le lampadaire éclaire plutôt que là où il est susceptible de les avoir égarées, le politologue a souvent tendance à lire les événements politiques avec ses seuls outils parce qu'ils lui sont immédiatement disponibles, et non avec les approches les plus pertinentes. En l'espèce, nous pensons que la crise de formation gouvernementale qu'a connue la Belgique en 2010-2011 peut être abordée sous l'angle psychologique. Plus spécifiquement, nous tentons ici de considérer certaines dynamiques psychologiques du nationalisme flamand, à travers une exposition de ses manifestations durant la crise.

Mots-clefs : Nationalisme, Flandre, N-VA, psychologie politique, crise politique belge (2007-2012), Vamik Volkan.

[English] As Robert Jervis famously argued, to look at political events without the help of psychological theories is a "drunkard's search", a search whose "logic" is that of easiness and not of pertinence. In this paper, we make ours this statement and consider that the recent political crisis in Belgium has to be understood - among others, of course - in psychological terms. More precisely, we argue that the psychological dynamics of Flemish nationalism impede the good resolution of the enduring political instability that recurrently handicaps Belgium.

Key words: Flemish nationalism, N-VA, political psychology, Belgian political crisis (2007-2011), Vamik Volkan.

\section{Introduction}

Affirmer que le malaise politique profond que traverse actuellement la Belgique comporte une dimension psychologique est un truisme. Ce n'est cependant pas encore, au vu de la faible résonnance de cet aspect dans le débat public, un lieu 
commun. Décrire en quoi une crise - et cette crise de formation gouvernementale en particulier - ressort du champ psychologique, tracer certains des processus mentaux et comportementaux qui lui donnent une ampleur inhabituelle, n'est pourtant pas sans intérêt. Explorer les ressorts d'une crise ou d'un conflit à la lumière d'une littérature académique en psychologie sociale et cognitive de plus en plus significative - tant du point de vue de la quantité produite que de la qualité atteinte est en effet devenu, comme le disait déjà le politologue Ole Holsti voici plus de vingt ans, «plus qu'un luxe $»^{1}$. Le cas belge n'échappe pas à la règle.

Il existe évidemment de très nombreux angles d'approche psychologiques de la situation belge actuelle, tant la psychologie est une discipline fragmentée. Nous invitons le lecteur soucieux de mieux comprendre le politique à explorer les potentialités heuristiques d'analyses telles que celles proposées, notamment, par la psychologie de la catégorisation ${ }^{2}$, par celle des dynamiques intergroupes ${ }^{3}$, ou encore par celle des émotions ${ }^{4}$. Notre but ici n'est évidemment pas de conglomérer ces approches et de les adapter au cas belge, en un agrégat peu digeste. Plutôt, nous souhaitons éclairer divers épisodes politiques de cette année passée par un même éclairage, en l'occurrence une lecture psycho-sociale du nationalisme flamand ${ }^{5}$. Ce dernier pèse en effet lourd dans la détermination des positions politiques, comme en témoigne l'assise électorale du parti ouvertement nationaliste (la N-VA, NieuwVlaamse Alliantie) et, partant, son statut clef dans les négociations jusqu'à son éviction des discussions ${ }^{6}$. L'idée, plus précisément, est ici de mettre en relation des épisodes de la crise apparemment anecdotiques, plutôt que se pencher sur les événements généralement perçus comme réellement importants et immédiatement politiques, afin de mettre en avant un portrait global de la dynamique nationaliste présente en Flandre, particulièrement dans le chef de la N-VA.

Cet éclairage commun, cette grille de lecture, nous pouvons la tirer de l'ouvrage de Vamik Volkan, The need to have enemies and allies - From clinical practice to

11 L'article de Holsti fait partie d'un numéro spécial du journal Political Psychology, constitué d'articles programmatiques en psychologie politique.

${ }^{2}$ Nous orientons ici à la fois vers l'approche cognitive, avec les travaux cruciaux de George Lakoff notamment, et vers l'approche sociale, intergroupe, avec par exemple les études de Samuel Gaertner, Penelope Oakes ou Stephen Reicher.

${ }^{3}$ Lire par exemple les influentes études d'Henri Tajfel et John Turner.

${ }^{4}$ Dans ce domaine, explorer les écrits de Jonathan Mercer ou, dans un autre esprit, ceux de Philippe Braud.

${ }^{5}$ Le nationalisme flamand est, bien entendu, un nationalisme régionaliste, qui n'est donc pas tourné vers l'État belge mais bien vers la Flandre.

${ }^{6}$ Cette éviction, basée sur un constat par certains partis traditionnels d'une volonté de blocage de la part du parti nationaliste, s'est opérée, il faut le reconnaître, en dépit du résultat clair des urnes. 
international relationship. La question centrale du livre est celle - que les négociateurs belges pourraient bien saisir à titre personnel - de savoir pourquoi certains conflits intergroupes défient tout effort de résolution. S'appuyant à la fois sur sa pratique clinique de psychologue et psychanalyste, sur ses recherches académiques dans le champ de la psychologie sociale et politique, et, par endroits, sur diverses sources extérieures parfois inhabituelles ${ }^{1}$, l'auteur - Chypriote - livre une analyse qui semble s'appliquer presque par magie au cas flamand. Les deux concepts centraux sont le traumatisme et le rituel. C'est en effet, soutient l'auteur, en examinant les traumatismes originels spécifiques d'un groupe et les rituels que ce dernier met en œuvre (avec ou sans succès, là est tout l'enjeu) pour dépasser ces traumatismes, que se comprend un nationalisme et ses tendances plus ou moins fortes à l'exclusion.

Évidemment, une telle grille de lecture pourrait tout autant être amenée pour rendre compte du nationalisme belge, dont il serait intéressant d'examiner les traumatismes et les rituels actuels. Il est à cet égard intéressant de constater que de nombreux intellectuels francophones, répondent aux manifestations de nationalisme flamand par un recours au patriotisme belge - plutôt que wallon, ou francophone. Ainsi lorsque certains responsables du tourisme en Flandre mettaient récemment en avant le label «Flanders remember» pour désigner les commémorations des batailles de la Première Guerre mondiale, certaines réactions ont surgi pour souligner le caractère unitaire belge de ces épisodes, plutôt que d'en appuyer par exemple le caractère tout autant wallon. Il reste que le nationalisme flamand, dont le principal (mais pas exclusif, comme nous allons le voir) thuriféraire est la N-VA, garde une position politique aujourd'hui nettement moins anecdotique, et accueille presque parfaitement, tel un calque, la grille de lecture de Volkan. ${ }^{2}$

\section{Traumatismes choisis}

L'analyse de Volkan se présente donc en deux pôles. Selon lui, tout groupe humain partage d'une part une série de traumatismes («traumas») qu'il considère comme cruciaux dans son histoire, et met d'autre part en place des « rituels» («rituals») destinés à dépasser ces traumatismes - souvent mais pas toujours, ces rituels s'appuient sur des «gloires choisies» («chosen glories»). L'idée est que le nationalisme émerge de l'incapacité du groupe à réellement dépasser, à positivement faire le deuil («mourning») des événements vécus collectivement comme des

\footnotetext{
${ }^{1}$ Tel un essai méconnu de George Orwell, « Notes on Nationalism » (1945).

${ }^{2}$ Nous simplifions ici les positions de l'auteur, le but étant de dresser un paysage, de proposer une ouverture, plutôt que d'avancer une analyse poussée des mécanismes sous-tendant les différents éléments donnés ici.
} 
traumatismes. En d'autres termes, la propension au nationalisme naîtrait d'une incapacité du groupe à la résilience, d'une utilisation contre-productive de la ritualisation. Au lieu de considérer les événements traumatiques comme appartenant au passé, de les comprendre par une perspective historique rigoureuse, le groupe exagère la continuité chronologique et structure sa compréhension du présent par un constant effort de référence au passé «présentifié » ${ }^{1}$ et de recherche des coupables des événements traumatiques :

«Le groupe tire une représentation mentale d'un événement traumatique au cœur de son identité. Il passe cette représentation mentale de cet événement - en même temps que les sentiments de blessure et de honte qui y sont associés, et les stratégies de défense contre les conflits perçus comme résultant de l'événement de génération en génération. » $(\mathrm{p} . \mathrm{xxv})^{2}$

A l'évidence, une série d'événements historiques ont une dimension traumatique pour certains Flamands, et prennent immédiatement une dimension quasimythologique: la discrimination linguistique face au feu de l'ennemi durant la Première Guerre mondiale, l'organisation linguistique du travail et du pouvoir caractérisant la révolution industrielle en Belgique, les privilèges de la langue française dans le système institutionnel belge avant les premières réformes linguistiques, sont trois exemples clairs. La mémoire de ces «événements »-dont la réalité historique est immanquablement boiteuse - reste très vive parmi les nationalistes flamands. En miroir, certains épisodes se sont nécessairement érigés en gloires, elles aussi soumises à interprétation quasi-mythique, comme bien sûr la Bataille des éperons d'or (1302), ressassée selon une lecture binaire comme une victoire des Flamands besogneux face aux riches aristocrates français.

Durant ces passages intergénérationnels de la mémoire collective, donc du sentiment de préjudice, l'exigence de vérité sur le(s) traumatisme(s) et sur le(s) gloire(s) forcément s'efface, laissant place à une lecture, une interprétation intouchable sous peine de débat moral :

«La vérité historique concernant l'événement n'importe plus; le rôle central que

l'événement joue au sein de l'identité ethnique du groupe devient plus important.

[...] À chaque génération, le récit se modifie; reste le rôle que l'événement possède, psychologiquement, dans l'identité du groupe. » (p.xxvi)

Ainsi, les lectures pour le moins simplistes et présentifiées de la de la Bataille des éperons d'or comme conflit entre pauvres Flamands et riches francophones, ou celles des abus de pouvoir des officiers francophones de 1914-1918, ou encore de l'exploitation francophone des Flamands au $19^{\text {ème }}$ siècle, ont en commun de contreplaquer les groupes d'aujourd'hui sur des clivages bien réels mais

${ }^{1}$ C'est-à-dire décontextualisé, analysé avec des classifications conceptuelles adaptées au présent mais qui ne font pas sens par rapport aux réalités, aux catégories passées.

${ }^{2}$ Cette citation, comme toutes les suivantes, est notre traduction. 
incommensurables d'époques révolues, faisant fi du hiatus chronologique et de ses conséquences sur les structures sociales réelles de la société. Le même scénario est à chaque fois rejoué, avec les mêmes acteurs. Chaque débat sur ces questions entraine, inévitablement, des débats où l'exigence historique cède à la position politique, rendant impossible toute communication rationnelle (dans le sens, par exemple, d'un Habermas ayant certainement sous-estimé les mécanismes psychologiques de la raison). En attestent, durant la crise actuelle, les sorties médiatiques de Mr. De Wever - chairman de la N-VA - sur la collaboration nazie des Flamands et des francophones, puis le caractère moral du débat qui s'est ensuivi.

L'indifférence à la vérité en vient en réalité à dépasser le seul domaine des tabous que sont devenus les traumatismes et gloires choisies, pour affecter tout jugement vis-à-vis d'interprétations a priori banales que l'autre groupe porte sur des événements parfois déconnectés des thématiques centrales du conflit intergroupe. Non pas que le groupe nationaliste en possède une lecture bien spécifique - les aurait déjà « codé », comme le dirait Deleuze -; il s'agit simplement, selon Volkan, d'une attitude d'accusation de parti-pris, de mauvaise foi. L'auteur donne l'exemple, très éclairant pour le cas belge, des médias :

«De cette indifférence à la vérité objective résulte un point de vue particulièrement subjectif vis-à-vis des nouvelles rapportées par les médias : tout dépendra alors de l'alignement du journaliste avec un côté ou avec l'autre, et de son appartenance à un groupe national ou ethnique ou à un autre. » (p.86)

Les rapports pour le moins difficiles, et c'est un euphémisme, que la N-VA a entretenu (en particulier au printemps 2011) et continue d'entretenir avec les médias belges francophones (spécialement Le Soir) voire français - comme en atteste l'échange acerbe survenu entre Messieurs Quatremer (blogueur journaliste à Libération) et Overmeer (porte-parole de la N-VA) - sont une illustration parfaite de cette incommensurabilité migrant des sujets traumatiques vers les autres à l'occasion d'une crise. Moins immédiate, mais tout aussi significative est la récurrence des accusations de lobbying francophone à l'étranger dès lors qu'une organisation internationale ou un média étranger pose une lecture sévère des politiques flamandes d'assimilation. Enfin, l'incompréhension mutuelle, les réprimandes acerbes sur la couverture francophone des funérailles de Mme Marie-Rose Morel, sont un dernier exemple $^{1}$. Évidemment, il n'est pas question d'affirmer ici que les médias francophones posent un regard objectif sur l'actualité flamande ou internationale -

\footnotetext{
1 Mme Morel, décédée d'un cancer alors que la N-VA était toujours à la table des négociations gouvernementales, était tout à la fois une figure populaire en Flandre (une bekende vlaaming) et une cadre du parti d'extrême-droite Vlaams Belang. Ses funérailles ont drainé un large public, ce qui a consterné une partie de la population francophone.
} 
là n'est pas la question. Le point est de montrer que quoi qu'écrivent ou disent ces médias, la posture d'accusation subsiste.

Mais la désignation d'un coupable du traumatisme, son érection en Autre si différent, s'accompagne d'un paradoxe - «plus nous essayons de nous distancer de l'ennemi [l'auteur a une acception très large du terme], plus grande devient notre préoccupation excessive vis-à-vis de ce dernier, et plus étroitement nous devenons liés à lui, consciemment et inconsciemment »(p.5). La mobilisation constante des traumatismes et des gloires par un groupe, la mise en avant des différences avec l'autre groupe, augmentent l'inévitabilité de se penser par rapport, en contraste avec ce dernier, et empêche le groupe de se penser seul.

\section{Rituels}

Les problèmes intergroupes surviennent donc dès lors que le processus de deuil («mourning », à comprendre dans son sens positif de dépassement, de résilience, de travail sur soi) de traumatismes anciens n'est pas conduit correctement par un groupe, dérape. Dans ce processus, les rituels occupent, selon Volkan, un rôle clef, et peuvent prendre diverses formes - ne se limitant pas à des commémorations, des défilés et autres manifestations. L'auteur indique qu'ils peuvent prendre des formes plus subtiles, comme l'élaboration d'une théorie politique partagée, des habitudes collectives, ou la tenue de groupes de discussion stratégiques. Chez les nationalistes flamands, le rassemblement de l'Y $\operatorname{ser}^{1}$ constitue toujours le rituel le plus visible, mais tend peut-être à perdre de l'importance par rapport à d'autres. L'issue de Bruxelles étant au cœur de la crise belge, le gordel, randonnée cycliste dont le parcours ceinture symboliquement Bruxelles pour montrer la continuité territoriale flamande, garde un rôle privilégié. Toujours dans le domaine sportif, la distribution de drapeaux flamands ou flamingants le long des courses cyclistes est également un rituel : en atteste la présence sur la page d'accueil du site web de la N-VA d'une photo bariolée d'étendards flamands de la célèbre course cycliste du Tour des Flandres (Ronde van Vlaanderen). Mais au-delà de ces manifestations évidentes, il peut être argumenté que la pérennisation active, répétée, de diverses théories, comme par exemple l'hypothèse de la «tache d'huile» francophone ${ }^{2}$ ou la

\footnotetext{
1 Ce rassemblement entend commémorer les combattants de la Première Guerre mondiale, et de ce fait participe à la continuation du mythe de l'inégalité linguistique face au feu de l'ennemi.

${ }^{2}$ La théorie de la «tâche d'huile » dénonce l'expansion territoriale des francophones sur le territoire flamand en périphérie de Bruxelles.
} 
«doctrine Maddens ${ }^{1}$, voire les récurrents «bons mots» (intelligents autant qu'acerbes) de Mr. De Wever, constituent aujourd'hui un rituel important et suivi.

Si de tels rituels ne sont pas de nature à permettre la construction d'une identité autonome (sans référence obligée à un Autre), ni à contribuer à la résilience du traumatisme, c'est qu'ils ont toujours, tels certains médicaments, cette double potentialité positive et négative, curative ou létale. Ainsi, tandis que certains rituels de groupe règlent le problème, d'autres au contraire l'aiguisent, attisent les rancœurs, laissent apparaître « des indices reconnaissables de douleur et de colère [...], continuant à réintroduire le passé dans le présent » (p.171), et contribuent au passage intergénérationnel du traumatisme et à la lecture mythologique de ce dernier. Une récente étude interuniversitaire - et intercommunautaire, précisons-le de psychologie sociale quantitative semble d'ailleurs apporter un élément de preuve à l'égard d'un tel sentiment de colère présent derrière la ritualisation dans le chef des responsables et votants de la N-VA. Examinant les émotions ressenties par des personnes s'identifiant à différents partis vis-à-vis de l'autre groupe linguistique, l'étude fait ressortir que les trois émotions dominantes dans les groupes de citoyens d'orientation N-VA sont, dans l'ordre, la colère, le ressentiment, et ensuite seulement le respect, une différence nette par rapport à tous les autres partis ${ }^{2}$. Ce résultat, particulièrement la prégnance de la colère et du ressentiment, qui sont des émotions très particulières en politique ${ }^{3}$, cadre parfaitement avec l'analyse de Volkan.

Dans ce contexte émotionnel, il ne peut être question d'humour ou d'autodérision, tant «la création de blagues constitue le début du processus de résilience du groupe » (p.xxvii). L'altercation entre Messieurs Geluck et E. Van Rompuy, sur le plateau de l'émission politique phare Mise au point du 24 octobre 2010, puis les défenses parallèles de ces derniers dans Le Soir du 26 octobre suivant, sont à cet égard tout sauf anecdotiques. L'épisode illustre, une fois de plus, l'enjeu central de la dynamique nationaliste, à savoir l'échec ou la réussite dans le processus de résilience d'événements traumatiques par une série de rituels sains. Mr. Toussaint, dans une chronique écrite quelques jours après les événements («Peut-on rire avec

\footnotetext{
${ }^{1}$ La « doctrine Maddens », du nom d'un professeur d'université, soutient qu'il n'est pas actuellement nécessaire pour les Flamands de négocier avec les francophones car la situation économique, à leur désavantage, les forcera un jour à mendier une négociation dans une situation extrêmement défavorable.

${ }^{2}$ Dans tous les autres groupes politiques en effet, le duo des émotions dominantes est le respect suivi de la sympathie - à l'exception notable du FDF, où la peur supplante le respect.

${ }^{3}$ Lire à ce sujet les travaux d'Élisabeth Meur, qui examine l'impact du ressentiment sur les dynamiques de raisonnement politique en situation de conflit.
} 
Van Rompuy ? »), avait raison d'insister, malgré toute l'emphase exagérée de sa prose, sur le fait que "le rire désinfecte, le rire cautérise, le rire cicatrise ». De nombreux observateurs ont remarqué qu'aux célébrations de victoire de la N-VA des dernières élections, Mr. De Wever n'avait pas souri. L'homme, qui ne manque pourtant pas d'humour, ne peut pas plaisanter sur certains thèmes - dont la gravité objective est pourtant loin d'égaler celle de l'holocauste, celle des inégalités profondes caractérisant les modes de production et de redistribution actuels, ou celle des grandes tensions interreligieuses actuelles.

Pour Volkan, les rituels ne présentent pas, s'ils sont correctement pratiqués, dans une configuration positive de dépassement de l'événement traumatique, de dimension «altruiste », c'est-à-dire de référence continue et insistance à un autre groupe. À l'inverse, dans le scénario nationaliste, ils présentent cette caractéristique, et doublement. D'abord via une dimension, purificatrice ( $«$ le groupe essaye d'éliminer tous les éléments étrangers afin de constituer une nouvelle identité cohérente et source de cohésion, qui est appelée à remplacer la précédente, qui était blessée» p.xxxi), et ensuite via une dimension réclamatrice («nous avons suffisamment été blessés - nous devons maintenant recevoir ce que les autres nous doivent » p.xxx). La dimension réclamatrice s'exprime très nettement en Flandre, par exemple dans le discours politique de la NVA. Économiquement orienté, celuici est sous-tendu par l'exigence d'une certaine forme de réparation par rapport à l'inégalité des transferts nord-sud, trouvant prétexte dans une raison qui se veut purement arithmétique, donc juste ${ }^{2}$. La présentation du wallon fainéant, la métaphore du «junkie » dans un Der Spiegel de décembre dernier, illustrent bien cette mise en avant dans un rituel (en l'occurrence celui des «bons mots» du leader emblématique) d'une «collection des traits que le groupe ne souhaite pas avoir » (p.6). En ce qui concerne la présence d'une dimension purificatrice, le constat est immédiatement hautement discutable. Tandis que les outils théoriques sont nombreux pour attester la différence d'ampleur qui sépare une politique publique communautarienne d'une autre véritablement raciste/ethniciste, on sait aussi, au moins depuis les travaux de Michel Foucault ${ }^{3}$, toute la continuité «biopolitique » entre ces deux pôles. Dans ce contexte théorique difficile et sensible sur lequel nous nous défendrons ici de prendre parti, certains éléments ambigus attestent du moins d'une propension purificatrice : le wooncode ${ }^{4}$, le refus de nommer les bourgmestres

\footnotetext{
${ }^{1}$ Dans Le Soir du 29 décembre 2010.

2 Comparer cette forme de justice à celle sous-tendue par une idée forte de la solidarité.

${ }^{3}$ Lire les dernières pages du cours de 1976 de Foucault au Collège de France, « Il faut défendre la société ».

${ }^{4}$ Règlement qui conditionne l'attribution de logements sociaux aux habitants parlant effectivement le néerlandais, ou étant inscrits à des cours intensifs de cette langue.
} 
du rand ${ }^{1}$, ou encore l'interdiction d'afficher en français dans certaines communes de la périphérie bruxelloise sont autant d'événements illustrant, au moins, la présence d'une tension dans la conception de soi des Flamands.

Pourquoi, enfin, une telle orientation négative dans le processus de deuil, dans la construction de l'identité de groupe? Pourquoi, en un mot, un nationalisme flamand en essor aujourd'hui ? La réponse de Volkan est commune, et somme toute assez simpliste. Elle tient en un mot - la peur, dans une situation de stress : «lors de contextes stressants, les rituels sont intensifiés par le groupe » (p.xxix). A l'échelle du globe, la situation globale est sans aucune contestation stressante, pour des raisons qui sont inutiles à rappeler ici. Tous les groupes politiques d'une certaine taille et portant un certain désir d'organisation politique sont confrontés à ce stress dû à l'érosion des frontières traditionnelles. Mais le stress est aussi, plus immédiatement, présent à l'échelle du pays. L'incertitude autant que la volonté de sécuriser un accord vu comme acceptable prédominent depuis les élections, et peuvent même être vues comme des éléments inhérents au cadre institutionnel instable de la particratie belge. Cette idée, presque biologisante, de stress n'est pas sans résonner avec l'interprétation, autrement plus provocatrice, de Greenfeld ${ }^{2}$, pour qui le nationalisme, comme exigence si profondément moderne d'égalité parfaite parmi les membres d'une société stablement et clairement identifiée, irait jusqu'à produire dans un monde qui ne permet plus, de facto, ces caractéristiques, nulle autre chose qu' une confusion relevant du trouble mental.

\section{Conclusions}

Miscevic, dans sa notice du Stanford Encyclopedia of Philosophy, définit le nationalisme de la façon suivante :

«(1) L'attitude que les membres d'une nation prennent lorsqu'ils prennent au sérieux [«care »] leur identité nationale, et (2) les actions que les membres d'une nation prennent lorsqu'ils cherchent à atteindre (ou à maintenir) l'autodétermination. »

La grille de lecture donnée par Volkan a le mérite de montrer que ces deux pôles sont intermêlés, que les attitudes (ci-dessus en [1]) impactent directement les actions (en [2]). Cette même grille de lecture permet de mettre en lumière divers événements de la crise belge actuelle qui sont bien loin d'être aussi déconnectés que leur apparence ne le laisse croire. Le contenu des «bons mots » récurrents de $\mathrm{Mr}$.

\footnotetext{
${ }^{1}$ Une polémique d'interprétation juridique est à l'origine du refus par les autorités de tutelle flamandes de valider la nomination des bourgmestres (maires) francophones élus dans certaines municipalités dites «à facilités» - sur sol flamand mais peuplées en majorité de francophones, qui y disposent de droits linguistiques élargis.

${ }^{2}$ Lire son article « Nationalism and the mind ».
} 
De Wever, l'altercation entre Messieurs Geluck et Van Rompuy, les tensions entre les médias francophones et la N-VA, le malaise autour des funérailles de Mme. Morel, la lecture binaire simpliste que donne Mr. De Wever de la collaboration nazie en Belgique, à un moment incongru, ne sont pas des anecdotes qui entourent les événements «plus importants» comme le serait tel ou tel changement de position politique dans les négociations, ou l'arrivée ou le départ de tel ou tel parti à la table. Bien du contraire, ce sont ces «anecdotes» qui, ensemble, fournissent la trame centrale de la crise actuelle, expliquant pourquoi le « conflit chronique devient de plus en plus difficile à résoudre, devient partie intégrante de l'identité d'un groupe ou d'une nation » (p.6).

Une telle lecture des événements a l'avantage de se détacher d'une lecture purement stratégique de la crise, pour en saisir, dans un mouvement plus heuristique, certaines des raisons. Il existe à l'évidence bien d'autres éclairages, et celui de Volkan est contestable à plusieurs égards. Mais son mérite reste double : d'une part il fournit une approche globale, et d'autre part il ne disqualifie pas normativement le nationalisme d'entrée de jeu, comme s'il s'agissait d'une irrationalité, d'une idéologie du passé. Le regard est plus sympathique - tout groupe cultive une ambiguité nationaliste dans sa mémoire collective et ses rituels - mais le diagnostic est par contre bien plus grave - le nationalisme est un problème dans le développement sain d'un groupe, qui peut avoir des répercussions profondes dans l'ouverture à l'Autre.

Examiner les dynamiques du nationalisme, a fortiori du seul nationalisme flamand, est loin d'être le seul regard heuristique sur la crise. Une telle approche est, de plus, souvent suspectée de condescendance. S'agissant de ce deuxième obstacle, nous pensons bien avoir montré que l'approche mobilisée ici dépasse le jugement normatif facile pour faire ressortir les propensions nationalistes de n'importe quel groupe. Vis-à-vis du premier problème, nous espérons que l'approche proposée ici s'est avérée suffisamment séduisante que pour encourager le lecteur à l'approfondir et à renouveler sa conception de la crise et de ses éventuels efforts de règlement.

\section{Références bibliographiques}

BRAUD P. (1996): Les émotions en politique: Problèmes d'analyse. Paris: Presses de Science Po.

DRUCKMAN D. (1994): "Nationalism, patriotism, and group loyalty: A social psychological perspective ». In Mershon International Studies Review, vol.38 $\mathrm{n}^{\circ} 1, \mathrm{pp} .43-68$.

FouCAult M. (1997): «Il faut défendre la société ». Cours au Collège de France, 1976. Paris: Gallimard - Seuil - Hautes Études. 
GREENFELD L. (2005): «Nationalism and the mind ». In Nations and Nationalism, vol.11 n³, pp.325-351.

Holsti O. (1989): «The political psychology of international politics: More than a luxury ». In Political Psychology, vol.10 n³, pp.495-500.

KYMLICKA W. (1995): The rights of minority culture. Oxford: Oxford University Press.

LAKOFF G. (1987): Women, fire, and dangerous things. What categories reveal about the mind. Chicago: The University of Chicago Press.

LAKOFF G. (2002): Moral Politics. How liberals and conservatives think. Chicago: The University of Chicago Press.

MERCER J. (2005): «Rationality and psychology in International politics ». In International Organization, vol.59 n 1 , pp.77-106.

Mesquita B., Delvaux E., Klein O., licata L., Mercy A., Rime B. (2010): Émotions et attitudes face aux relations entre les communautés linguistiques en Belgique chez des répondants francophones et néerlandophones de différentes orientations politiques. Rapport interuniversitaire et intercommunautaire en ligne, disponible sur http://www.psycho-psysoc.site.ulb.ac.be/images/stories/ oklein/EtudeBHVsyntheseprovisoire.pdf.

MEUR E. (2011): «Resentment and security. Affective origins of regional security complex ». Présentation à International Studies Association Annual Convention, Montreal, mars 2011.

MisCEVIC N. (2010): «Nationalism ». In Stanford Encyclopedia of Philosophy. Notice encyclopédique en ligne, disponible sur http://plato.stanford.edu/ entries/nationalism/.

OAKES P. (2001): "The root of all evil in intergroup relations? Unearthing the categorization process ». In BROWN R., GAERTNER S., ed.: Intergroup Processes, pp.3-21. Oxford: Blackwell.

REICHER S., HOPKINS N. (2001): « Psychology and the end of history: A critique and proposal for the psychology of social categorization ». In Political Psychology, vol. $22 \mathrm{n}^{\circ} 2$, pp.383-407.

TAJFEL H., TURNER J. (1979) : «An integrative theory of intergroup conflict ». In AUSTIN W., WORCHEL S., ed.: The social psychology of intergroup relations, pp.33-48. Monterey CA: Brooks.

VOLKAN V. (1994): The need to have enemies and allies. From clinical practice to international relationships. Northvale NJ: Jason Aronson. 
WALZer M. (1984): Spheres of justice. A defense of pluralism and equality. NewYork: Basic Books.

Walzer M. (1989): Nation and universe. The Tanner Lectures on Human Values. Exposés en ligne, disponibles sur http://www.tannerlectures.utah.edu/ lectures/documents/walzer90.pdf. 